\title{
Introduction to Topological Insulators
}

\author{
Hongming Weng, Xi Dai, Zhong Fang \\ Beijing National Laboratory for Condensed Matter Physics, \\ and Institute of Physics, Chinese Academy of Sciences, Beijing 100190, China;
}

(Dated: Jul. 5, 2011)

\begin{abstract}
In this article, we will give a brief introduction to the topological insulators. We will briefly review some of the recent progresses, from both theoretical and experimental sides. In particular, we will emphasize the recent progresses achieved in China.

PACS numbers:
\end{abstract}




\section{INTRODUCTION.}

Topological insulator (TI) has drawn extensive attentions recently in the field of condensed matter physics, not only because of its fundamental importance but also because of its potential applications for future technology. One of the most simple and distinct properties of TIs is that it is electronically insulating in bulk while conducting along its boundary (for examples, edges in two dimensional systems and surfaces in three dimensional systems), due to the topologically unavoidable gapless boundary states. The existence of metallic boundary states is very robust, and they are protected by topological invariants. Though this property is in sharp contrast to our general knowledge on normal insulators or simple metals, similar phenomena has been observed and well-known since 1980, due to the discovery of quantum Hall effect (QHE) [1]. In QHE, electrons in two-dimensional (2D) materials are enforced to change its quantum state into a new one, so called Landau energy level, under highly intensive magnetic field. The original free-electron-like conducting electrons start to make cyclotron motion. It is easy to understand that in the bulk of the material, full circle cyclotron motion leads to electron localization, resulting in an insulating bulk. While along the edge of the 2D system, the circle motion of electrons enforced by the magnetic field can not be completed due to the presence of edge, which make the electrons travel in a way forming so called edge state, and electrons in such state can circumambulate defects or impurities on their way "smartly" (due to the absence of back scatterings). Therefore, current carried by these electrons is dissipationless and conductance is quantized into unit of $e^{2} / \hbar$ with quantum number corresponding to the number of edge states. Such fascinating quantum state and physical phenomena are highly interesting and impact the whole field of physics. Since then, people started to realize that this is a new state of quantum matters and it should be characterized by topology of electronic wavefunctions. This topological number can be evaluated from the formula given by D. J. Thouless, M. Kohmoto, M. P. Nightingale and M. den Nijs [2]. It is called as TKNN number or first Chern number. This topological number has direct physical meaning and is experimentally observable, because it is the same as the number of edge states or the quantum number of Hall conductance.

Obviously the application of QHE requires highly intensive magnetic field and low temperature. In 1988, F. D. M. Haldane [3] proposed a genius model to realize the quantum Hall state in solid state materials without introducing Landau levels. His model is based on 
graphene like honeycomb lattice structure, which has two sublattices. If equal and opposite magnetic fluxes can be threaded through these two sublattices, respectively, the electrons hopping at least upto next nearest neighbor within such lattice model would be in a quantum state similar to the quantum Hall state. While the possible materials realizing Haldane's dream is still under intensive pursuing upto today, on the other hand, in 2005, C. L. Kane and E. J. Mele [4] made another big step forward to reach Haldane's dream. They demonstrated that spin-orbit coupling, which is inherent in any material, can effectively play a role of the imagined magnetic field in Haldane's lattice model. Different from the quantum Hall state, however, the time reversal symmetry is preserved in the later case. As C. L. Kane and E. J. Mele pointed out, the electrons within such graphene model are in another new quantum state, characterized by a topological invariant $\mathrm{Z}_{2}$ number, which is also determined by the topology of wavefunctions describing this state. Electrons in this state behave in the similar way as in QHE, namely they are insulating in the bulk but conducting on the edges. Insulator possessing this kind of quantum state is coined as "topological insulator" by J. Moore and L. Balents [5]. From the non-interacting single particle picture, in the bulk of TIs, the occupied electron-bands are well separated from the unoccupied electron-bands by a energy gap at Fermi level. However, due to the bands "twisting", the occupied states are topologically non-trivial, and contribute to a non-zero $\mathrm{Z}_{2}$ integer number. If there are edges or interfaces formed between two insulators with different $Z_{2}$ number, there must appear conducting edge states. The insulators which has "twisted" bands are therefore very much different from normal insulator or vacuum with "untwisted" bands. Compared with QHE, 2D TI has at least two edge states for each edge, due to the time reversal symmetry. The electrons in two edge stats must have opposite spins, and they must travel in opposite directions. This is exactly the quantized spin Hall effect [6], therefore, 2D TI is also called as quantum spin Hall insulator.

Though graphene is predicted as a prototype 2D TI, Y. Yao et al. found that the spinorbit coupling in graphene is negligibly small [7] and pointed out that the bulk insulating gap is too small to observe quantum spin Hall effect. In 2006, B. A. Bernevig, T. A. Hughes and S. C. Zhang [9] theoretically predicted that the HgTe quantum well can be tuned into topological insulating phase and its edge states can give out quantum spin Hall effect. They also established an intuitive model hamiltonian to describe the topological phase transition driven by "band inversion". Soon in 2007, quantum spin Hall effect shows 
up in HgTe quantum well insulator just as predicted in the experiment done by M. König et al. [10]. And a detailed tight-binding model study on HgTe is given by X. Dai et al. [11]. Recently, C. C. Liu et al. proposed that Silicene [8] with low-buckled sheet structure with enhanced spin-orbit coupling might show detectable quantum spin Hall effect, which might be suitable for applications in spintronic devices since it is compatible with current siliconbased microelectronic industry.

\section{SEARCHING FOR THREE-DIMENSIONAL TOPOLOGICAL INSULATORS.}

Nearly at the same time in 2006, L. Fu et al. [12], J. Moore et al. [5], as well as R. Roy [13], extended the concept of TIs to three-dimensional (3D) case. Similar as 2D HgTe quantum well, 3D $\mathrm{Bi}_{1-x} \mathrm{Sb}_{x}$ alloy is predicted [12, 14, 15] by theoretical calculations to have topological nontrivial conducting surface state depending on $x$ value and then it is confirmed by experimental measurements [16], though the topologically protected surface state is complex and the bulk band gap is too small for possible applications. The important realization of 3D TIs are tetradymite semiconductors $\mathrm{Bi}_{2} \mathrm{Se}_{3}, \mathrm{Bi}_{2} \mathrm{Te}_{3}$ and $\mathrm{Sb}_{2} \mathrm{Te}_{3}$, which are, again, predicted by theoretical calculations [17] and confirmed by experimental measurements [18, 19]. This new family 3D TIs have single Dirac cone like surface states and bulk band gap is as larger as $0.3 \mathrm{eV}$, which simplifies the further study on the properties of surface states and promises the potential applications even under room temperature [20]. After this, lots of efforts have been performed to find various 3D TIs. B. Yan et al. [21] and H. Lin et al. [22] have independently predicted that thallium-based III-V-VI $\mathrm{VI}_{2}$ ternary chalcogenides might be 3D TIs, and later experimental works done by T. Sato et al. [23] and K. Kuroda et al. [24] have confirmed their predictions. Some other ternary half-Heusler compounds [25 27], chalcopyrite compounds [29] and materials with antiperovskite structure [28], as well as honeycomb lattice [30], have been predicted to be 3D TI with or without additional uniaxial strain. Recently, another new binary TI $\mathrm{Ag}_{2}$ Te has been proposed by W. Zhang et al. [31], which has highly anisotropic Dirac cone surface state, in contrast to known examples. Therefore, there are plenty of materials having nontrivial topological state. They supply a solid material basis for studying various intriguing physical properties of surface states, as well as potential applications. Theoretical predictions based on first-principles calculations have played a crucial important role in searching for new TIs, which accelerates the study of this 
field tremendously.

The defining property of TIs is the topologically protected surface state, which is spinmomentum locked, and $\pi$ Berry phase is acquired for the wavefunctions circling the Dirac cone. Angle-resolved photoemission spectroscopy (ARPES) is the usual and widely used method to directly observe this surface state [16, 18, 19, 23, 24]. To detect the chiral spin texture of surface state, as well as $\pi$ Berry phase, one useful technique is the spin-resolved ARPES, which is firstly applied on $\mathrm{Bi}_{1-x} \mathrm{Sb}_{x}$ alloy [32] and then on $\mathrm{Bi}_{2} \mathrm{Se}_{3}[33], \mathrm{Bi}_{2} \mathrm{Te}_{3}$ [34, 35] and $\mathrm{TlBiSe}_{2}[34]$. When surface states are close to the bulk states, they start to deform from the circle-like Dirac cone and follow the bulk crystal symmetry. The spin texture will be modified at the same time. Such effect is demonstrated by ARPES or spin-resolved ARPES for $\mathrm{Bi}_{2} \mathrm{Te}_{3}[19,34-36]$, where Fermi level is systematically tuned by carrier doping. This effect has been explained well by an effective model [37] and reproduced by first-principles calculations [31, 38, 39].

The spin-momentum locked conducting surface state is predicted to have one important feature: the conducting electron will not be back-scattered or localized by non-magnetic (time reversal symmetric) scattering potential. One evidence of this feature is the measurement of interference patterns on the surface of TI by scanning tunneling spectroscopy. T. Zhang et al. [40] confirm that the backscattering of surface states is absent on non-magnetic $\mathrm{Ag}$ doped $\mathrm{Bi}_{2} \mathrm{Te}_{3}$ (111) surface. P. Roushan et al. [41] show that the atomic disorder in $\mathrm{Bi}_{1-x} \mathrm{Sb}_{x}$ will not cause backscattering of surface states. Y. Okada et al. [42] demonstrate that magnetic impurity $\mathrm{Fe}$ doped $\mathrm{Bi}_{2} \mathrm{Te}_{3}$ breaks time reversal symmetry and the backscattering does exist. Nevertheless, the direct transport measurements of surface conducting states are hindered since the residual conduction in the bulk from impurities or defects in sample can easily overcome the surface contributions. H. Peng et al. [43] circumvent this problem by preparing $\mathrm{Bi}_{2} \mathrm{Te}_{3}$ nanoribbon, which has much large surface/volume ratio. They observed Aharonov-Bohm interference for the currents conducting through the surface of TI nanoribbon. J. Chen et. al. [44] successfully grow $\mathrm{Bi}_{2} \mathrm{Se}_{3}$ on $\mathrm{SrTiO}_{3}$ substrates, which enables very large tunablity in carrier density with a back gate. They observed weak antilocalization (WAL) effect in magnetoresistance measurements, which originates from the $\pi$ Berry phase obtained by surface electrons circling the Dirac cone. H.-T. He et al. [45] further demonstrated that the non-magnetic impurity will not influence the WAL effect while the magnetic impurity quenches the WAL effect in $\mathrm{Bi}_{2} \mathrm{Te}_{3}$. M. Liu et al. [46] have done 
the similar experiments on $\mathrm{Cr}$ doped $\mathrm{Bi}_{2} \mathrm{Se}_{3}$. The direct evidence that surface states can overcome the non-magnetic lattice imperfection is the experiment done by J. Seo et al. [47] on Sb surface, where they observed that the topological surface states can penetrate the atomic steps instead of being reflected or absorbed as in common metals.

\section{OPENING GAP ON SURFACE STATES: TOWARDS APPLICATIONS}

Many of the potential applications of TIs are related to the gap opening of topological surface states. Some of the possibilities will be discussed in this section. Since time reversal symmetry is conserved in 3D TI, there should be two Dirac cones according to fermion doubling theorem [48]. They are in fact located on the opposite surfaces, which are well separated by the insulating bulk. W. Zhang et al. [38] has shown that in $\mathrm{Bi}_{2} \mathrm{Se}_{3}$ the surface states can penetrate into bulk by about $4 \mathrm{~nm}$. Therefore, if film sample is thin enough, the two surface states can have finite coupling and a gap would open at Dirac cone point. C.-X. Liu et al. [49] have pointed out that there will be an oscillatory crossover from 2D to 3D TI as film thickness increases. This crossover behavior has been observed by ARPES experiments performed on $\mathrm{Bi}_{2} \mathrm{Se}_{3}[50,51]$ and $\mathrm{Bi}_{2} \mathrm{Te}_{3}$ [52].

In addition to the coupling between two surface states, magnetic impurities which break time reversal symmetry can also open a gap at the Dirac point of surface states. Fe ions have been introduced into $\mathrm{Bi}_{2} \mathrm{Se}_{3}$ [53], and ARPES experiment has observed the opening of gap. Mn and Fe ions have also been introduced into $\left.\mathrm{Bi}_{2} \mathrm{Te}_{3}, 54,55\right]$ and similar ARPES experiments have confirmed the gap opening. R. Yu et al. [56] have proposed that ferromagnetic TI can be obtained in $\mathrm{Cr}$ and $\mathrm{Fe}$ doped thin film $\mathrm{Bi}_{2} \mathrm{Se}_{3}$ and quantum anomalous Hall effect can be realized in this way. When perpendicular external magnetic field is applied onto the surface state, Landau level will appear. The Landau level for Dirac electrons is different from that for the parabolic free electrons. There is one zero energy Landau level independent of magnetic field strength B. Other Landau level energy is proportional to $\sqrt{B}$ [57]. P. Chen et al. [58] and T. Hanaguri et al. [58] have observed such Landau quantization of surface states in $\mathrm{Bi}_{2} \mathrm{Se}_{3}$ independently by scanning tunneling microscopy and spectroscopy. This kind of Landau level is also the basis for theory of quantum magnetoresistance proposed by A. A. Abrikosov [60], which is used to explain the observed non-saturated linear magnetoresistance in non-magnetic $\mathrm{Ag}_{2} \mathrm{Te}[31]$. 
Introducing time-reversal-breaking effects into TI can lead to topological magnetoelectric effect. The electromagnetic response of magnetic TI is the same as axion electrodynamics. Therefore, the search for and study of axion insulator becomes another important direction in sense of finding large magnetoeletric coupling materials. $\mathrm{Na}_{2} \mathrm{IrO}_{3}[61]$ is firstly proposed to be a TI, which contains $5 d$ transition metal possessing competing electron-electron correlation interaction $U$ and spin-orbit coupling. D. Pesin and L. Balents [62] then suggest that certain iridium based pyrochlore compounds such as $\operatorname{Pr}_{2} \operatorname{Ir}_{2} \mathrm{O}_{7}$ may be 3D TIs. X. Wan et al. [63] further studied pyrochlore $\mathrm{Y}_{2} \mathrm{Ir}_{2} \mathrm{O}_{7}$ by first-principles calculations. They found rich phase diagram of $\mathrm{Y}_{2} \mathrm{Ir}_{2} \mathrm{O}_{7}$ as electron-electron correlation interaction $U$ changes, including the topologically nontrivial axion insulator, Weyl semimetal, etc. The Weyl semimetal phase has intriguing fermi arc as its surface states, although this phase is sensitive to the parameter $U$ used in their calculations and might not be the nature state of the material. X. Wan et al. [64] further predicted that hypothetical Osmium spinel compounds such as $\mathrm{CaOs}_{2} \mathrm{O}_{4}$ and $\mathrm{SrOs}_{2} \mathrm{O}_{4}$ might have the similar phase diagram depending on $U$. Recently, G. Xu et al. [65] find that spinel $\mathrm{HgCr}_{2} \mathrm{Se}_{4}$ is a Weyl semimetal in its natural ferromagnetic ground state. The $\mathrm{HgCr}_{2} \mathrm{Se}_{4}$ is in fact a so called "Chern semi-metal", because the Weyl fermions are realized at the topological phase boundary separating the different insulating layers (in momentum $\vec{k}$ space) with different Chern numbers, where the Weyl fermions are topologically unavoidable. It is further proposed that the quantum anomalous Hall effect is a direct observable effect in quantum well of $\mathrm{Hg}_{2} \mathrm{CrSe}_{4}$, which might be confirmed by future experiments.

Besides these, breaking gauge symmetry due to proximity to a superconductor can also open a gap on topological surface states. L. Fu and C. L. Kane [66] proposed that the proximity effect between an $s$-wave superconductor and the surface states will result in a $2 \mathrm{D}$ state resembling a spinless $p_{x}+i p_{y}$ superconductor without breaking time reversal symmetry. This state supports Majorana bound states at vortices. Majorana fermions are particles which are their own antiparticles [67]. They constitute only half of a usual fermion, and obey the non-Abelian statistics [68], which is the key ingredient for the fault-tolerant topological quantum computation [69]. The experimental setting can be in principles obtained from laboratory, however, since most known 3D TIs are not good bulk insulators and important surface states may overlap with bulk states, experiments have to wait for development of well-controlled clean samples. On the other hand, a well matched interface between TI and $s$-wave superconductor is required to get large proximity effect. The discovery of high 


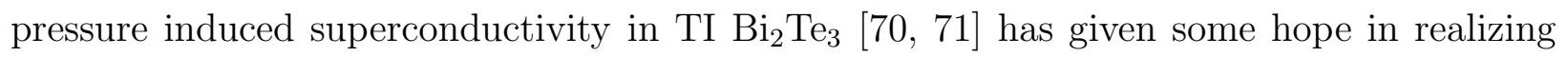
topological superconductivity in one compound to overcome this problem. It was also proposed that semiconductor quantum wells with Rashba type spin-orbit coupling in proximity to $s$-wave superconductor will produce similar effect [72 74]. This may lower the experimental threshold, since well-controlled samples are available nowadays. These proposals are encouraging, while experimental obstacles still remain. First, magnetic insulating layers or strong external magnetic field are required to break the time reversal symmetry, which is not easy to implement experimentally; second, the Fermi surfaces in both cases are too small, and fine control of chemical potential is difficult for semiconductors in contact with a superconductor. Using half-metal with odd number Fermi surfaces instead of TI or semiconductor quantum well is then proposed [75-77] to overcome the above difficulties. The asymmetric interface between half-metal and $s$-wave superconductor induces Rashba type spin-orbit coupling as the case using semiconductor quantum well. H. Weng et al. [78] have proposed that $\mathrm{NaCoO}_{2}$ is the right material to realize this. They find that $\mathrm{NaCoO}_{2}$ itself is insulating while its surface is half-metalic and has just one single large Fermi surface. The exchange splitting is as large as $0.2 \mathrm{eV}$, which simplifies the tuning of chemical potential. These make it a promising candidate for experimental setting up to find Majorana fermion.

[1] K. Von Klitzing, G. Dorda and M. Pepper, Phys. Rev. Lett. 45, 494 (1980).

[2] D. J. Thouless, M. Kohmoto, M. P. Nightingale and M. den Nijs, Phys. Rev. Lett. 49, 405 (1982).

[3] F. D. M. Haldane, Phys. Rev. Lett. 61, 2015 (1988).

[4] C. L. Kane, E. J. Mele, Phys. Rev. Lett. 95, 226801 (2005), C. L. Kane, E. J. Mele, Phys. Rev. Lett. 95, 146802 (2005).

[5] J. E. Moore, L. Balents, Phys. Rev. B 75, 121306(2007).

[6] B. A. Bernevie, and S. C. Zhang, Phys. Rev. Lett. 96, 106802 (2006).

[7] Y. Yao, F. Ye, X. L. Qi, S. C. Zhang, Z. Fang, Phys. Rev. B 75, 041401 (2007).

[8] Cheng-Cheng Liu, Wanxiang Feng, and Yugui Yao, e-print arXiv:1104.1290 (2011).

[9] B. A. Bernevig, T. A. Hughes, S. C. Zhang, Science 314, 1757 (2006).

[10] M. König, S. Wiedmann, C. Brne, A. Roth, H. Buhmann, L. W. Molenkamp, X. L. Qi and S. 
C. Zhang, Science 318, 766 (2007).

[11] Xi Dai, Taylor L. Hughes, Xiao-Liang Qi, Zhong Fang, Shou-Cheng Zhang, Phys. Rev. B 77, 125319 (2008).

[12] L. Fu, C. L. Kane, E. J. Mele, Phys. Rev. Lett. 98, 106803 (2007).

[13] Rahul Roy, Phys. Rev. B 79, 195332 (2009).

[14] S. Murakami, New J. Phys. 9, 356 (2007).

[15] H. J. Zhang, C. X. Liu, X. L. Qi, X. Y. Deng, X. Dai, S. C. Zhang, Z. Fang, Phys. Rev. B 80, 085307 (2009).

[16] D. Hsieh, D. Qian, L. Wray, Y. Xia, Y. S. Hor, R. J. Cava, M. Z. Hasan, Nature 452, 970 (2008).

[17] H. J. Zhang et al., Nature Phys. 5, 438 (2009).

[18] Y. Xia et al., Nature Phys. 5, 398 (2009).

[19] Y. L. Chen, J. G. Analytis, J.-H. Chu, Z. K. Liu, S.-K. Mo, X. L. Qi, H. J. Zhang, D. H. Lu, X. Dai, Z. Fang, S. C. Zhang, I. R. Fisher, Z. Hussain, Z.-X. Shen, Science 325, 178 (2009).

[20] Joel Moore, Nature Phys. 5, 378 (2009).

[21] B. Yan, C.-X. Liu, H.-J. Zhang, C.-Y. Yam, X.-L. Qi, T. Frauenheim, S.-C. Zhang, Europhys. Lett. 90, 37002 (2010).

[22] H. Lin, R. S. Markiewicz, L. A. Wray, L. Fu, M. Z. Hasan, and A. Bansil, Phys. Rev. Lett. 105, $036404(2010)$.

[23] Takafumi Sato, Kouji Segawa, Hua Guo, Katsuaki Sugawara, Seigo Souma, Takashi Takahashi, and Yoichi Ando, Phys. Rev. Lett. 105, 136802 (2010).

[24] K. Kuroda, M. Ye, A. Kimura, S. V. Eremeev, E. E. Krasovskii, E. V. Chulkov, Y. Ueda, K. Miyamoto, T. Okuda, K. Shimada, H. Namatame, and M. Taniguchi, Phys. Rev. Lett. 105, 146801 (2010).

[25] Stanislav Chadov, Xiaoliang Qi, Jürgen Kübler, Gerhard H. Fecher, Claudia Felser and Shou Cheng Zhang, Nature Materials 9, 514 (2010).

[26] Hsin Lin, L. Andrew Wray, Yuqi Xia, Suyang Xu, Shuang Jia, Robert J. Cava, Arun Bansil, M. Zahid Hasan, Nature Materials 9, 546 (2010).

[27] Di Xiao, Yugui Yao, Wanxiang Feng, Jun Wen, Wenguang Zhu, Xing-Qiu Chen, G. Malcolm Stocks, and Zhenyu Zhang, Phys. Rev. Lett. 105, 096404 (2010).

[28] Y. Sun, X. Chen, S. Yunoki, D. Li, Y. Li, Phys. Rev. Lett. 105, 216406 (2010). 
[29] Wanxiang Feng, Di Xiao, Jun Ding, and Yugui Yao, Phys. Rev. Lett. 106, 016402 (2011).

[30] Hai-Jun Zhang, Stanislav Chadov, Lukas Müchler, Binghai Yan, Xiao-Liang Qi, Jürgen Kübler, Shou-Cheng Zhang, and Claudia Felser, Phys. Rev. Lett. 106, 156402 (2011).

[31] Wei Zhang, Rui Yu, Wanxiang Feng, Yugui Yao, Hongming Weng, Xi Dai, Zhong Fang, Phys. Rev. Lett. 106, 156808 (2011).

[32] D. Hsieh, Y. Xia, L. Wray, D. Qian, A. Pal, J. H. Dil, J. Osterwalder, F. Meier, G. Bihlmayer, C. L. Kane, Y. S. Hor, R. J. Cava, M. Z. Hasan, Science 323, 919 (2009).

[33] D. Hsieh, Y. Xia, D. Qian, L. Wray, J. H. Dil, F. Meier, J. Osterwalder, L. Patthey, J. G. Checkelsky, N. P. Ong, A. V. Fedorov, H. Lin, A. Bansil, D. Grauer, Y. S. Hor, R. J. Cava, and M. Z. Hasan, Nature 460, 1101 (2009).

[34] S. Souma, K. Kosaka, T. Sato, M. Komatsu, A. Takayama, T. Takahashi, M. Kriener, Kouji Segawa, and Yoichi Ando, Phys. Rev. Lett. 106, 216803 (2011).

[35] Su-Yang Xu, L. A. Wray, Y. Xia, F. von Rohr, Y. S. Hor, J. H. Dil, F. Meier, B. Slomski, J. Osterwalder, M. Neupane, H. Lin, A. Bansil, A. Fedorov, R. J. Cava, M. Z. Hasan, e-print arXiv:1101.3985 (2011).

[36] Guang Wang, Xie-Gang Zhu, Yi-Yang Sun, Yao-Yi Li, Tong Zhang, Jing Wen, Xi Chen, Ke He, Li-Li Wang, Xu-Cun Ma, Jin-Feng Jia, Shengbai B. Zhang, and Qi-Kun Xue, Adv. Mater. 23, n/a. (2011). doi: 10.1002/adma.201100678

[37] L. Fu, Phys. Rev. Lett. 103, 266801 (2009).

[38] W. Zhang, R. Yu, H. Zhang, X. Dai, Z. Fang, New J. Phys. 12, 065013 (2010).

[39] Hai-Jun Zhang, Chao-Xing Liu, Xiao-Liang Qi, Xiao-Yu Deng, Xi Dai, Shou-Cheng Zhang, and Zhong Fang, Phys. Rev. B 80, 085307 (2009).

[40] Tong Zhang, Peng Cheng, Xi Chen, Jin-Feng Jia,1 Xucun Ma, Ke He, Lili Wang, Haijun Zhang, Xi Dai, Zhong Fang, Xincheng Xie, and Qi-Kun Xue, Phys. Rev. Lett. 103, 266803 (2009).

[41] Pedram Roushan, Jungpil Seo, Colin V. Parker, Y. S. Hor, D. Hsieh, Dong Qian, Anthony Richardella, M. Z. Hasan, R. J. Cava, Ali Yazdani, Nature 460, 1106 (2009).

[42] Yoshinori Okada, Chetan Dhital, Wenwen Zhou, Erik D. Huemiller, Hsin Lin, S. Basak, A. Bansil, Y.-B. Huang, H. Ding, Z. Wang, Stephen D. Wilson, and V. Madhavan, Phys. Rev. Lett. 106, 206805 (2011).

[43] Hailin Peng, Keji Lai, Desheng Kong, Stefan Meister, Yulin Chen, Xiao-Liang Qi, Shou-Cheng 
Zhang, Zhi-Xun Shen, and Yi Cui, Nature Mater. 9, 225 (2010); Thomas Ihn, Nature Mater. 9, 187 (2010).

[44] J. Chen, H. J. Qin, F. Yang, J. Liu, T. Guan, F. M. Qu, G. H. Zhang, J. R. Shi, X. C. Xie, C. L. Yang, K. H. Wu, Y.Q.Li, and L.Lu, Phys. Rev. Lett. 105, 176602 (2010).

[45] Hong-Tao He, Gan Wang, Tao Zhang, Iam-Keong Sou, George K. L Wong, Jian-Nong Wang, Hai-Zhou Lu, Shun-Qing Shen, and Fu-Chun Zhang, Phys. Rev. Lett. 106, 166805 (2011).

[46] Minhao Liu, Jinsong Zhang, Cui-Zu Chang, Zuocheng Zhang, Xiao Feng, Kang Li, Ke He, Li-li Wang, Xi Chen, Xi Dai, Zhong Fang, Qi-Kun Xue, Xucun Ma, and Yayu Wang, e-print arXiv:1103.3353 (2011).

[47] Jungpil Seo, Pedram Roushan, Haim Beidenkopf, Y. S. Hor, R. J. Cava, Ali Yazdani, Nature 466, 343 (2010); Marcel Franz, Nature 466, 323 (2010).

[48] H. Nielssen and N. Ninomiya, Phys. Lett. 130B, 389 (1983).

[49] Chao-Xing Liu, HaiJun Zhang, Binghai Yan, Xiao-Liang Qi, Thomas Frauenheim, Xi Dai, Zhong Fang, and Shou-Cheng Zhang, Phys. Rev. B 81, 041307(R) (2010).

[50] Yi Zhang, Ke He, Cui-Zu Chang, Can-Li Song, Li-Li Wang, Xi Chen, Jin-Feng Jia, Zhong Fang, Xi Dai, Wen-Yu Shan, Shun-Qing Shen, Qian Niu, Xiao-Liang Qi, Shou-Cheng Zhang, Xu-Cun Ma and Qi-Kun Xue, Nature Phys. 6, 584 (2010).

[51] Yusuke Sakamoto, Toru Hirahara, Hidetoshi Miyazaki, Shin-ichi Kimura, and Shuji Hasegawa, Phys. Rev. B 81, 165432 (2010).

[52] Y.-Y. Li, G. Wang, X.-G. Zhu, M.-H. Liu, C. Ye, X. Chen, Y.-Y. Wang, K. He, L.-L. Wang, X.-C. Ma, H.-J. Zhang, X. Dai, Z. Fang, X.-C. Xie, Y. Liu, X.-L. Qi, J.-F. Jia, S.-C. Zhang, and Q.-K. Xue, e-print arXiv:0912.5054 (2009).

[53] Y. L. Chen, J.-H. Chu, J. G. Analytis, Z. K. Liu, K. Igarashi, H.-H. Kuo, X. L. Qi, S. K. Mo, R. G. Moore, D. H. Lu, M. Hashimoto, T. Sasagawa, S. C. Zhang, I. R. Fisher, Z. Hussain, Z. X. Shen, Science 329, 659 (2010).

[54] Y. S. Hor, P. Roushan, H. Beidenkopf, J. Seo, D. Qu, J. G. Checkelsky, L. A. Wray, D. Hsieh, Y. Xia, S.-Y. Xu, D. Qian, M. Z. Hasan, N. P. Ong, A. Yazdani, and R. J. Cava, Phys. Rev. B 81, 195203 (2010).

[55] L. Andrew Wray, Su-Yang Xu, Yuqi Xia, David Hsieh, Alexei V. Fedorov, Yew San Hor, Robert J. Cava, Arun Bansil, Hsin Lin and M. Zahid Hasan, Nature Phys. 7, 32 (2011); Eli Rotenberg, Nature Phys. 7, 8 (2011). 
[56] Rui Yu, Wei Zhang, Hai-Jun Zhang, Shou-Cheng Zhang, Xi Dai, Zhong Fang, Science 329, $61(2010)$.

[57] R. Jackiw, Phys. Rev. D 29, 2375 (1984); Y. Zheng and T. Ando, Phys. Rev. B 65, 245420 (2002).

[58] Peng Cheng, Canli Song, Tong Zhang, Yanyi Zhang, Yilin Wang, Jin-Feng Jia, Jing Wang, Yayu Wang, Bang-Fen Zhu, Xi Chen, Xucun Ma, Ke He, Lili Wang, Xi Dai, Zhong Fang, Xincheng Xie, Xiao-Liang Qi, Chao-Xing Liu, Shou-Cheng Zhang, and Qi-Kun Xue, Phys. Rev. Lett. 105, 076801 (2010).

[59] T. Hanaguri, K. Igarashi, M. Kawamura, H. Takagi, and T. Sasagawa, Phys. Rev. B 82, 081305(R) (2010).

[60] A. A. Abrikosov, Phys. Rev. B 58, 2788 (1998).

[61] A. Shitade, H. Katsura, J. Kunes, X. L. Qi, S. C. Zhang and N. Nagaosa, Phys. Rev. Lett. 102, $256403(2009)$.

[62] Dmytro Pesin and Leon Balents, Nature Phys. 6376 (2010).

[63] Xiangang Wan, Ari M. Turner, Ashvin Vishwanath, and Sergey Y. Savrasov, Phys. Rev. B 83, 205101 (2011); L. Balnets, Physics 4, 36 (2011).

[64] Xiangang Wan, Ashvin Vishwanath and Sergey Y. Savrasov, e-print arXiv:1103.4634 (2011).

[65] Gang Xu, Hongming Weng, Zhijun Wang, Xi Dai, Zhong Fang, e-print arXiv:1106.3125 (2011).

[66] L. Fu and C. L. Kane, Phys. Rev. Lett. 100, 096407 (2008).

[67] See, for example, F. Wilczek, Nature Phys. 5, 614 (2009), and references therein.

[68] C. Nayak et al. Rev. Mod. Phys. 80, 1083 (2008).

[69] A. Kitaev, Ann. Phys. (N.Y.), 303, 2 (2003).

[70] J. L. Zhanga, S. J. Zhang, H. M. Weng, W. Zhang, L. X. Yang, Q. Q. Liu, S. M. Feng, X. C. Wang, R. C. Yu, L. Z. Cao, L. Wang, W. G. Yang, H. Z. Liu, W. Y. Zhao, S. C. Zhang, X. Dai, Z. Fang, and C. Q. Jin, Proc. Natl. Acad. Sci. USA 108, 24 (2011).

[71] Chao Zhang, Liling Sun, Zhaoyu Chen, Xingjiang Zhou, Qi Wu, Wei Yi, Jing Guo, Xiaoli Dong, and Zhongxian Zhao, Phys. Rev. B 83, 140504(R) (2011).

[72] J. D. Sau, R. M. Lutchyn, S. Tewari, and S. Das Sarma, Phys. Rev. Lett. 104, 040502 (2010).

[73] J. Alicea, Phys. Rev. B 81, 125318 (2010).

[74] A. C. Potter et al., e-print arXiv:cond-mat/1011.6371 (2010).

[75] P. A. Lee, e-print arXiv:cond-mat/0907.2681 (2009). 
[76] S. B. Chung et al., e-print arXiv:cond-mat/1011.6422 (2010).

[77] M. Duckhein, P. W. Brouwer, Phys. Rev. B 83, 054513 (2011).

[78] Hongming Weng, Gang Xu, Haijun Zhang, Shou-Cheng Zhang, Xi Dai, Zhong Fang, e-print arXiv:cond-mat/1103.1930 (2011). 